\title{
Landau gauge condensates from global color model
}

\author{
Zhao Zhang $^{a, b, 1}$, Wei-qin Zhao ${ }^{a, b, 2}$ \\ ${ }^{a}$ CCAST(World Laboratory), P.O. Box 8730, \\ Beijing 100080, P. R. China \\ ${ }^{b}$ Institute of High Energy Physics, Academica Sinica, \\ Beijing, 100039, P. R. China
}

\begin{abstract}
We compute the dimension-2 gluon pair condensate $g^{2}\langle A A\rangle$ and the dimension-4 mixed quark-gluon condensate $\langle\bar{q} g A \cdot \gamma q\rangle$ in Landau gauge within the framework of global color model. The result for the dynamical gluon mass is within the range given by other independent determinations. The obtained mixed Landau gauge condensate $\langle\bar{q} g A \cdot \gamma q\rangle$ is clearly dependent on the definitions of the condensates. We show that the consistent result may be obtained when the same definitions are used.
\end{abstract}

Keywords: QCD vacuum ; Global color model; Dyson-Schwinger equations; Condensates; Mass gap

PACS number(s): 12.38.Lg; 11.15.Tk; 12.38.Aw; 12.39.-x

\footnotetext{
${ }^{1}$ E-mail address: zhangzh@ccastb.ccast.ac.cn

${ }^{2}$ E-mail address: chaowq@ccast.ac.cn
} 


\section{Introduction}

The vacuum condensates are believed to play an important role for characterizing the nonperturbative aspect of QCD. The well known gauge invariant condensates such as $\langle\bar{q} q\rangle$ and $\left\langle\alpha_{s} G^{2}\right\rangle$ are proved to be crucial in the application of SVZ sum rules. Recently, there has been growing evidence for the existence of the dimension 2 condensate $\left\langle A_{\mu}^{a} A_{\mu}^{a}\right\rangle$ in $S U\left(N_{c}\right)$ pure Yang-Mills theory and QCD. Lavelle and Schaden [1] was responsible for first introducing the non vanishing $\left\langle A_{\mu}^{a} A_{\mu}^{a}\right\rangle$ to study the gauge dependent Green functions by making use of the operator product expansion (OPE). The recent evidence for the importance of the condensate $\left\langle A_{\mu}^{a} A_{\mu}^{a}\right\rangle$ based on OPE can be found in [2, 3, 4]. The non-vanishing gluon pair condensate gives rise to the dynamical gluon mass which is determined by

$$
m_{g}^{2}=\frac{N_{c}}{\left(N_{c}^{2}-1\right) D}(\alpha+5-D)\left\langle g^{2} A_{\mu}^{a} A_{\mu}^{a}\right\rangle,
$$

where $\alpha$ stands for the Lorentz covariant gauge fixing parameter and $D$ is the space-time dimension.

Early implications of the gluon pair condensate have been explored by Celenza and Shakin [5]. Naively, the operator $A^{2}$ is not gauge invariant. The importance of the condensate raises the question of gauge invariance and leads to a number of papers which discuss how this condensate could play a role in gauge invariant formulation [6, 7, 8, 9, 10, Kondo [6] introduced a BRST invariant and Anti-BRST invariant combination of dimension 2 operator

$$
O=\frac{1}{\Omega} \int d^{D} x \operatorname{tr}\left[\frac{1}{2} A_{\mu}(x) A_{\mu}(x)-i \alpha C(x) \bar{C}(x)\right],
$$

where $C(x)$ and $\bar{C}(x)$ are the Faddeev-Popov ghost and $\Omega$ is the volume of the D-dimensional space-time. The vacuum expectation value $(\mathrm{VEV})$ of this dimension 2 operator reduces to the gluon condensate in Landau gauge with $\alpha=0$ which has a definite physical meaning [7] .

The role of the dimension 4 mixed quark gluon condensate $\left\langle g \bar{q} \frac{\lambda^{a}}{2} A_{\mu}^{a} \lambda^{\mu} q\right\rangle$ has been studied in 11, 12] in the Lorentz covariant gauge and it has been shown that this condensate has the contributions to the quark propagator and the gluon propagator. This mixed condensate is clearly gauge non-invariant. Recently, the authors of Ref. 4] have evaluated the values of the gluon pair condensate and the dimension 4 mixed condensate from the quenched lattice results for the quark propagator in the Landau gauge and claimed that it is the first determination of the mixed condensate $\left\langle g \bar{q} \frac{\lambda^{a}}{2} A_{\mu}^{a} \lambda^{\mu} q\right\rangle$. This has motivated us to investigate both of these Landau gauge condensates from the Global Color Model.

\section{Formalism}

The Global Color Model(GCM) is a quark-gluon filed theory with the action

$$
S_{G C M}\left[\bar{q}, q, A_{\mu}^{a}\right]=\int\left(\bar{q}\left(\gamma \cdot \partial+m-i g A_{\mu}^{a} \frac{\lambda^{a}}{2} \gamma_{\mu}\right) q+\frac{1}{2} A_{\mu}^{a} D_{\mu \nu}^{-1}(i \partial) A_{\nu}^{a}\right)
$$


and the generating functional

$$
Z[J, \bar{\eta}, \eta]=\int D \bar{q} D q D A \exp \left(-S_{G C M}\left[\bar{q}, q, A_{\mu}^{a}\right]+\bar{\eta} q+\bar{q} \eta+J_{\mu}^{a} A_{\mu}^{a}\right) .
$$

The essence of GCM is that it models the QCD local gluonic action $\int F_{\mu \nu}^{a} F_{\mu \nu}^{a}$ which has local color symmetry, by a highly nonlocal action which has a global color symmetry. To quote Cahill and Gunner [13: "There is an Infrared(IR) saturation effect which in conjunction with the dynamical breaking of chiral symmetry, appears to suppress details of the formal color gauge symmetry of QCD." The main aspects of GCM have been reviewed in [13, 14, 15.

In Euclidean metric, with $\left\{\gamma_{\mu}, \gamma_{\nu}\right\}=2 \delta_{\mu \nu}$ and $\gamma_{\mu}^{+}=\gamma_{\mu}$, the inverse of the dressed quark propagator in the chiral limit has the decomposition

$$
G^{-1}(p)=i \gamma \cdot p+\Sigma(p)=i \gamma \cdot p A\left(p^{2}\right)+B\left(p^{2}\right)
$$

where $\Sigma(p)$ stands for the dressing self-energy of quarks. Within the GCM formalism, the quark self-energy is determined by the rainbow-ladder truncated quark DSE

$$
\Sigma(p)=\frac{4}{3} \int \frac{d^{4} q}{(2 \pi)^{4}} g^{2} D_{\mu \nu}(p-q) \gamma_{\mu} G(q) \gamma_{\nu}
$$

where $g^{2} D_{\mu \nu}(p-q)$ is the effective gluon propagator. In Landau gauge with $g^{2} D_{\mu \nu}(p)=$ $\left(\delta_{\mu \nu}-\frac{p_{\mu} p_{\nu}}{p^{2}}\right) g^{2} D\left(p^{2}\right)$, the nontrivial solution to Eq. (6) which is characterized by $B\left(p^{2}\right) \neq 0$ is determined by the two coupled integrable functions

$$
\begin{aligned}
\left(A\left(p^{2}\right)-1\right) p^{2} & =\frac{4}{3} \int \frac{d^{4} q}{(2 \pi)^{4}} g^{2} D(p-q)\left(p \cdot q+2 \frac{q \cdot(p-q) p \cdot(p-q)}{(p-q)^{2}}\right) \frac{A\left(q^{2}\right)}{q^{2} A^{2}\left(q^{2}\right)+B^{2}\left(q^{2}\right)}(7) \\
B\left(p^{2}\right) & =4 \int \frac{d^{4} q}{(2 \pi)^{4}} g^{2} D(p-q) \frac{B\left(q^{2}\right)}{q^{2} A^{2}\left(q^{2}\right)+B^{2}\left(q^{2}\right)}
\end{aligned}
$$

while the trivial solution to Eq. (6) which is characterized by $B\left(p^{2}\right)=0$ is determined by

$$
\left(A^{\prime}\left(p^{2}\right)-1\right) p^{2}=\frac{4}{3} \int \frac{d^{4} q}{(2 \pi)^{4}} g^{2} D(p-q)\left(p \cdot q+2 \frac{q \cdot(p-q) p \cdot(p-q)}{(p-q)^{2}}\right) \frac{1}{q^{2} A^{\prime}\left(q^{2}\right)} .
$$

Within the GCM formalism, the "Nambu-Goldstone" phase is described by the nontrivial quark propagator since chiral symmetry is dynamically broken with the no-vanishing constituent quark mass $M\left(p^{2}\right)=B\left(p^{2}\right) / A\left(p^{2}\right)$ in the chiral limit and the dressed quarks are confined since it does not have a Lehmann representation. The "Wigner" phase is described by the corresponding trivial quark propagator $G^{\prime}(p)=\frac{1}{i \gamma \cdot p A^{\prime}\left(p^{2}\right)}$ with neither chiral symmetry breaking nor confinement. The quark propagators $G(x-y)$ and $G^{\prime}(x-y)$ can be seen as the expectation values of the operator $T q(x) \bar{q}(y)$ over the physical vacuum state $|V\rangle$ and the perturbative vacuum state $|P\rangle$ respectively within GCM formalism. 
Since the functional integration over the gluon field $A_{\mu}^{a}$ is quadratic according to (6), one can perform the integration over gluon field analytically. Taking the quark color current $j_{\mu}^{a}=$ $i g \bar{q} \frac{\lambda^{a}}{2} \gamma_{\mu} q$ as the external source term for the gluon field $A_{\mu}^{a}$, we have the typical gaussian integrations

$$
\begin{aligned}
\int \mathcal{D} A e^{-\frac{1}{2} A D^{-1} A+j A} & =e^{\frac{1}{2} j D j} \\
\int \mathcal{D} A A e^{-\frac{1}{2} A D^{-1} A+j A} & =(j D) e^{\frac{1}{2} j D j} \\
\int \mathcal{D} A A^{2} e^{-\frac{1}{2} A D^{-1} A+j A} & =\left[D+(j D)^{2}\right] e^{\frac{1}{2} j D j} .
\end{aligned}
$$

Through above technique, the gluons vacuum averages are replaced by the quark color current $j_{\mu}^{a}$ together with the effective gluon propagator $D$. This method is very similar to the determination of the VEVs of the QCD operators involving the gluon fields in the instanton liquid model, where by integrating over the instanton coordinates, one derives an effective quark action of the form of a Nambu-Jona-Lasinio model [16]. This method was first used by Meissner [17. to calculate the gauge invariant dimension 5 mixed quark-gluon condensate $\langle g \bar{q} \sigma G q\rangle$ in GCM. In the mean field level, it is straightforward to calculate the vacuum expectation value (VEV) of any quark operator of the form

$$
\mathcal{O}_{n} \equiv\left(\bar{q}_{j_{1}} \Lambda_{j_{1} i_{1}}^{(1)} q_{i_{1}}\right)\left(\bar{q}_{j_{2}} \Lambda_{j_{2} i_{2}}^{(2)} q_{i_{2}}\right) \cdots\left(\bar{q}_{j_{n}} \Lambda_{j_{n} i_{n}}^{(n)} q_{i_{n}}\right),
$$

where $\Lambda^{(i)}$ stands for an operator in Dirac, color and flavor space. The expression for the VEV of the operator $\mathcal{O}_{n}$ takes the form

$$
\left\langle\mathcal{O}_{n}\right\rangle=(-1)^{n} \sum_{p}(-)^{p}\left[\Lambda_{j_{1} i_{1}}^{(1)} \cdots \Lambda_{j_{n} i_{n}}^{(n)} G_{i_{1} j_{p(1)}} \cdots G_{i_{n} j_{p(n)}}\right]
$$

where $p$ stands for the permutation of the $n$ indexes. According to (12), one can obtain the familiar expression for the quark condensate

$$
\langle\bar{q} q\rangle=-\frac{3}{4 \pi^{2}} \int_{0}^{\infty} d s s \frac{B(s)}{s A^{2}(s)+B^{2}(s)} .
$$

The expression for the four quark condensate $\left\langle\bar{q} \Lambda^{(1)} q \bar{q} \Lambda^{(2)} q\right\rangle$ can also be obtained within this formalism which is consistent with the result based on the vacuum saturation approximation.

Note that there is UV divergence in the calculation of the vacuum condensates based on Eq.(12). The UV divergence of Eq.(12) can be traced back to the UV behavior of both the scalar quark self energy function $B\left(p^{2}\right)$ and the vector quark self energy function $A\left(p^{2}\right)$. The ultraviolet behavior of the constituent quark mass $M\left(p^{2}\right)=B\left(p^{2}\right) / A\left(p^{2}\right)$ takes the form in the Landau gauge 18

$$
M\left(p^{2}\right)=\frac{2 \pi^{2} \gamma_{m}}{3} \frac{-\langle\bar{q} q\rangle^{0}}{p^{2}\left(\frac{1}{2} \ln \left[\frac{p^{2}}{\Lambda_{Q C D}^{2}}\right]\right)^{1-\gamma_{m}}}
$$


where $\gamma_{m}=12 /\left(33-2 N_{f}\right)$ is the mass anomalous dimension, with $N_{f}$ the number of light-quark and $\langle\bar{q} q\rangle^{0}$ the renormalization-group-invariant condensate. It is easy to see that the Eq.(13) is logarithmic divergent according to the UV behavior of $B\left(p^{2}\right) / A\left(p^{2}\right)$. In Ref [19], the quark condensate is well-defined and the UV divergence problem of the quark condensate is conquered by multiplying a renormalization constant. Within the framework of GCM, Eq. (13) will be free of UV divergence if the chosen effective gluon propagator has finite momentum range. Since the vacuum condensates are mainly determined by the low and medium momentum properties of the Schwinger functions of QCD, ignoring the effects from the hard gluonic radiative corrections will have little impact on the determination of these condensates. Note that the definition of the vacuum condensate in Ref [17], which used a finite cutoff on the integral, is incorrect since the obtained values for the quark condensate and the mixed quark-gluon condensate are distinctly sensitive to the cutoff. Without the cutoff, exploring of the thermal properties of $g\langle\bar{q} \sigma G q\rangle$ based on GCM formalism was made in Ref. [20].

Even in the case of not taking into account the asymptotic behavior of the effective gluon propagator, the UV behavior of the function $A(s)$ can also lead to the UV divergence problem in the calculation of the condensates based on Eq.(12). In Ref [21], the calculation of the mixed tensor susceptibility was performed based on (12) where the UV divergence caused by the UV behavior of the function $A(s)$ was cancelled by subtracting the corresponding perturbative contribution which was evaluated by the trivial quark propagator $G^{\prime}(p)$. The UV divergences induced by the function $A(s)$ also appear in the calculation of the Landau gauge condensates in this letter. Actually, this kind of divergence is due to no subtraction of the perturbative contribution in the calculation since in this case the Eq.(12) is also divergent if one substitutes $G^{\prime}(x-y)$ for $G(x-y)$. Because the condensates reflect the nonperturbative structure of QCD vacuum, the perturbative contribution to Eq.(12) must be subtracted. In the framework of GCM, the perturbative contribution can be self consistently evaluated by replacing the nontrivial quark propagator $G$ in Eq.(12) with the corresponding trivial quark propagator $G^{\prime}$. As a matter of fact, the trivial quark propagator has been used extensively to play the role of the perturbative dressed quark propagator in the study of thermal properties of QCD within the DSE formalism, where the bag constant was defined as the difference of the pressure between the true $Q C D$ vacuum and the perturbative QCD vacuum, which were evaluated by the Nambu-Goldstone solution and the Wigner solution to the quark propagator, respectively 22].

Therefore, the formula for the calculation of the VEV of operators in terms of quark fields should be changed by subtracting the corresponding trivial contribution

$$
\begin{aligned}
\left\langle: \mathcal{O}_{n}:\right\rangle= & (-1)^{n} \sum_{p}(-)^{p}\left[\Lambda_{j_{1} i_{1}}^{(1)} \cdots \Lambda_{j_{n} i_{n}}^{(n)} G_{i_{1} j_{p(1)}} \cdots G_{i_{n} j_{p(n)}}\right] \\
& -(-1)^{n} \sum_{p}(-)^{p}\left[\Lambda_{j_{1} i_{1}}^{(1)} \cdots \Lambda_{j_{n} i_{n}}^{(n)} G_{i_{1} j_{p(1)}}^{\prime} \cdots G_{i_{n} j_{p(n)}}^{\prime}\right]
\end{aligned}
$$


In fact, for the cases of the determination of the quark condensate and gauge invariant mixed quark gluon condensate, there are no needs to subtract these trivial terms which are rigorously zero due to $B\left(p^{2}\right) \equiv 0$.

Since the GCM is not renormalizable and the adopted effective gluon propagator in our calculation has a finite range in the momentum space, the scale at which a condensate is defined in our method is a typical hadronic scale, which is implicitly determined by the chosen effective gluon propagator $D(s)$ and the corresponding solution of the quark gap equations. In fact, the situation is very similar to the calculation of the condensates based on the instanton liquid model [16] where the the scale is set by the inverse instanton size.

\section{Evaluation of Landau gauge condensates}

With the above preparation, the gluon pair condensate and the dimension 4 mixed quarkgluon condensate can be evaluated in the framework of GCM. Using Eq.(10) and Eq.(15), we get the condensate $g^{2}\left\langle A_{\mu}^{a}(0) A_{\mu}^{a}(0)\right\rangle$

$$
\begin{aligned}
g^{2}\left\langle A_{\mu}^{a}(x) A_{\mu}^{a}(x)\right\rangle= & \left\langle g^{2} D_{\mu \mu}^{a a}(x-x)\right\rangle-\int d^{4} x^{\prime} \int d^{4} y^{\prime} g^{2} D_{\mu \mu^{\prime}}^{a a^{\prime}}\left(x-x^{\prime}\right) g^{2} D_{\mu \nu^{\prime}}^{a b^{\prime}}\left(x-y^{\prime}\right) \\
& \times\left\langle: \bar{q}\left(x^{\prime}\right) \gamma_{\mu^{\prime}} \frac{\lambda^{a^{\prime}}}{2} q\left(x^{\prime}\right) \bar{q}\left(y^{\prime}\right) \gamma_{\nu^{\prime}} \frac{\lambda^{b^{\prime}}}{2} q\left(y^{\prime}\right):\right\rangle
\end{aligned}
$$

where

$$
\begin{aligned}
\left\langle: \bar{q}\left(x^{\prime}\right) \gamma_{\mu^{\prime}} \frac{\lambda^{a^{\prime}}}{2} q\left(x^{\prime}\right) \bar{q}\left(y^{\prime}\right) \gamma_{\nu^{\prime}} \frac{\lambda^{b^{\prime}}}{2} q\left(y^{\prime}\right):\right\rangle= & \operatorname{tr}\left[\gamma_{\mu^{\prime}} \frac{\lambda^{a^{\prime}}}{2} G\left(x^{\prime}-y^{\prime}\right) \gamma_{\nu^{\prime}} \frac{\lambda^{b^{\prime}}}{2} G\left(y^{\prime}-x^{\prime}\right)\right] \\
& -\operatorname{tr}\left[\gamma_{\mu^{\prime}} \frac{\lambda^{a^{\prime}}}{2} G^{\prime}\left(x^{\prime}-y^{\prime}\right) \gamma_{\nu^{\prime}} \frac{\lambda^{b^{\prime}}}{2} G^{\prime}\left(y^{\prime}-x^{\prime}\right)\right] .
\end{aligned}
$$

From Eq.(16), one can see that the contribution to the gluon pair condensate is divided into two parts in the mean field level: the contribution from the effective propagator and the contribution from the four quark condensate. After Fourier transformation, we have

$$
\begin{aligned}
g^{2}\left\langle A_{\mu}^{a}(x) A_{\mu}^{a}(x)\right\rangle= & 24 \int \frac{d^{4} p}{(2 \pi)^{4}} g^{2} D\left(p^{2}\right)+4 \int \frac{d^{4} p}{(2 \pi)^{4}} \frac{d^{4} q}{(2 \pi)^{4}} g^{2} D(p-q) g^{2} D(p-q) \\
& \times\left\{12 B\left(p^{2}\right) B\left(q^{2}\right) Z\left(p^{2}\right) Z\left(q^{2}\right)+\left[4 p \cdot q+8 \frac{(p-q) \cdot p(p-q) \cdot q}{(p-q)^{2}}\right]\right. \\
& \left.\times\left[A\left(p^{2}\right) A\left(q^{2}\right) Z\left(p^{2}\right) Z\left(q^{2}\right)-A^{\prime}\left(p^{2}\right) A^{\prime}\left(q^{2}\right) Z^{\prime}\left(p^{2}\right) Z^{\prime}\left(q^{2}\right)\right]\right\} \\
= & \frac{3}{2 \pi^{2}} \int_{0}^{\infty} d s s g^{2} D(s)+\frac{1}{8 \pi^{5}} \int_{0}^{\infty} d s \int_{0}^{\infty} d t \int_{-1}^{1} d x s t \sqrt{1-x^{2}} \\
& \times\left[g^{2} D(s+t-2 x \sqrt{s t})\right]^{2}\left\{3 Z(s) Z(t) B(s) B(t)+\left[2 \frac{(s-\sqrt{s t} x)(\sqrt{s t} x-t)}{s+t-2 \sqrt{s t} x}\right.\right. \\
& \left.+\sqrt{s t} x]\left[Z(s) Z(t) A(s) A(t)-Z^{\prime}(s) Z^{\prime}(t) A^{\prime}(s) A^{\prime}(t)\right]\right\}
\end{aligned}
$$


with $Z\left(p^{2}\right)=1 /\left(A^{2}\left(p^{2}\right) p^{2}+B^{2}\left(p^{2}\right)\right)$ and $Z^{\prime}\left(p^{2}\right)=1 /\left(A^{\prime 2}\left(p^{2}\right) p^{2}\right)$.

Applying the same method, we obtain the expression for the mixed condensate in Landau gauge

$$
\begin{aligned}
-i g\left\langle\bar{q} A_{\mu}^{a} \frac{\lambda^{a}}{2} \gamma_{\mu} q\right\rangle= & -4 \int d^{4} x^{\prime} g^{2} D_{\mu \nu}\left(x-x^{\prime}\right)\left\{\operatorname{tr}_{D}\left[\gamma_{\mu} G\left(x-x^{\prime}\right) \gamma_{\nu} G\left(x^{\prime}-x\right)\right]\right. \\
& \left.-\operatorname{tr}_{D}\left[\gamma_{\mu} G^{\prime}\left(x-x^{\prime}\right) \gamma_{\nu} G^{\prime}\left(x^{\prime}-x\right)\right]\right\} \\
= & -12 \int \frac{d^{4} p}{(2 \pi)^{4}}\left\{\left[\frac{B^{2}\left(p^{2}\right)}{A^{2}\left(p^{2}\right) p^{2}+B^{2}\left(p^{2}\right)}+\frac{A\left(p^{2}\right)\left(A\left(p^{2}\right)-1\right) p^{2}}{A^{2}\left(p^{2}\right) p^{2}+B^{2}\left(p^{2}\right)}\right]\right. \\
& \left.-\left[\frac{A^{\prime}\left(p^{2}\right)-1}{A^{\prime}\left(p^{2}\right)}\right]\right\} \\
= & \frac{3}{4 \pi^{2}} \int_{0}^{\infty} d s s\left\{\frac{A(s) s}{\left(A^{2}(s) s+B^{2}(s)\right)}-\frac{1}{A^{\prime}(s)}\right\} .
\end{aligned}
$$

Note that the quark DSE

$$
\Sigma(x-y)=\frac{4}{3} g^{2} D_{\mu \nu}(x-y) \gamma_{\mu} G(x-y) \gamma_{\nu}
$$

has been used again in above derivation, which strongly simplifies the evaluation of (19). From (19), one can see that the mixed condensate vanishes when QCD undergoes a phase transition from the Nambu-Goldstone phase to the Wigner phase with $B \rightarrow 0$ and $A \rightarrow A^{\prime}$.

As mentioned above, the reduced version of Eqs.(18) and (19) without the corresponding subtraction term will be UV divergent. The UV divergence arises from the behavior of $A\left(p^{2}\right)$ at large momentum region. In the GCM formalism, $A\left(p^{2}\right)$ decreases with increasing $p^{2}$ until it takes the value $A\left(p^{2}\right)=1$ at $p^{2}=\infty$. This property can be illustrated by a simple analytic confining infrared-dominant (ID) model

$$
g^{2} D_{\mu \nu}(p-q)=(2 \pi)^{4} \frac{\eta^{2}}{4} \delta^{4}(p-q)\left(\delta_{\mu \nu}-\frac{p_{\mu} p_{\nu}}{p^{2}}\right)
$$

which was proposed in Ref [23]. The ID model has been used extensively in the literature, which can exhibit many of the qualitatively features based on more complicated ansantz. The Nambu-Goldstone solution for this model is

$$
\begin{aligned}
& A\left(p^{2}\right)=\left\{\begin{array}{cc}
2, & p^{2}<\frac{\eta^{2}}{4}, \\
\frac{1}{2}\left(1+\sqrt{1+\frac{2 \eta^{2}}{p^{2}}}\right), & \text { otherwise },
\end{array}\right. \\
& B\left(p^{2}\right)=\left\{\begin{array}{cc}
\sqrt{\eta^{2}-4 p^{2}}, & p^{2}<\frac{\eta^{2}}{4}, \\
0, & \text { otherwise. }
\end{array}\right.
\end{aligned}
$$

The corresponding Wigner solution takes the form

$$
B^{\prime}\left(p^{2}\right) \equiv 0, \quad A^{\prime}\left(p^{2}\right)=\frac{1}{2}\left(1+\sqrt{1+\frac{2 \eta^{2}}{p^{2}}}\right) .
$$


We can see that the function $A\left(p^{2}\right)$ is identical to $A^{\prime}\left(p^{2}\right)$ in the $p^{2}>\frac{\eta^{2}}{4}$ region and monotonously decreases with $p^{2}$ until it reaches 1 at $\infty$. Using the ID model, the $A\left(p^{2}\right)$ induced UV divergence without subtraction can be easily shown. For example, due to $A(s)-1 \sim \frac{1}{s}$ for $s \rightarrow \infty$ according to (22), the second term of right hand side of Eq.(19) is clearly divergent if we do not adopt the subtraction procedure. From Eq.(22) and Eq.(24), it is also shown that the contribution of the last term in Eq. (19) to the mixed condensate is mainly determined by the low energy behavior of the $A\left(p^{2}\right)$ and $A^{\prime}\left(p^{2}\right)$.

The chosen model ansatz for $g^{2} D(s)$ in this letter is the Gaussian type model

$$
g^{2} D_{\mu \nu}^{a b}(q)=4 \pi^{2} \Delta \frac{q^{2}}{\omega^{2}} \exp \left(-\frac{q^{2}}{\omega^{2}}\right) \delta^{a b} t_{\mu \nu}(q)
$$

where the parameter $\Delta$ sets the strength of the interaction and $\omega$ is the range parameter at which the scalar function in the parameterization is maximal. This Gaussian type form was supposed to represent a sensible hadron model in 24] and the parameter $\omega$ was envisaged to have a value of several hundred $\mathrm{MeV}$ which sets the interaction scale. In our work, these parameters are constrained by giving the reasonable constituent quark mass $M_{q}\left(q^{2}=0\right)$ and the pion decay constant fixed at $87 \mathrm{MeV}$ which is more appropriate in the chiral limit rather than the Pion's mass-shell value of $93 \mathrm{MeV}$. To check the sensitivity of our results on the model effective gluon propagator, various sets of parameters $\Delta$ and $\omega$ were used in our calculations.

Table 1. Calculated Landau gauge condensates from the Gaussian model in the chiral limit with three sets of parameters. The quark condensate and the constituent quark mass from this model are also listed. The units for $\Delta, \omega, M_{g}, M_{q}$ and $f_{\pi}$ are GeV.

\begin{tabular}{cccccccc}
\hline \hline$\Delta$ & $\omega$ & $\langle\bar{q} q\rangle\left(\mathrm{GeV}^{3}\right)$ & $g^{2}\langle A A\rangle\left(\mathrm{GeV}^{2}\right)$ & $g\langle\bar{q} A \cdot \gamma q\rangle\left(\mathrm{GeV}^{4}\right)$ & $M_{g}$ & $M_{q}(0)$ & $f_{\pi}$ \\
\hline 30.0 & 0.40 & $-0.215^{3}$ & 9.5 & $-1.2 * 10^{-3}$ & 0.94 & 0.381 & 0.087 \\
\hline 19.0 & 0.45 & $-0.223^{3}$ & 9.2 & $-1.3 * 10^{-3}$ & 0.93 & 0.342 & 0.087 \\
\hline 13.2 & 0.50 & $-0.232^{3}$ & 9.9 & $-1.4 * 10^{-3}$ & 0.96 & 0.311 & 0.087 \\
\hline \hline
\end{tabular}

Table 1 shows that the dynamical gluon mass obtained in the Gaussian model is around $1 \mathrm{GeV}$. This value is compatible with the estimates obtained by other approaches, which are within the range $0.5-1.5 \mathrm{GeV}$ [25]. Actually, from our numerical studies, the main contribution to the gluon pair condensate comes from the first term of right hand side of Eq.(16). This is shown in Table 2 with the dynamical gluon masses $M_{g 1}$ which is determined only from the first term of the Eq.(16). Comparing $M_{g 1}$ with $M_{g}$, we get the conclusion that the gluon pair condensate is mainly determined by the low energy part of $g^{2} D(p)$ and the correction from the four quark condensates can be neglected. This is reasonable since in GCM the $g^{2} D(p)$ is interpreted as the effective gluon propagator. Our results further confirms that the Gaussian model is surely an effective quark-quark interaction form in the low momentum region. In 
addition, this also provides a constraint to the form of $g^{2} D(p)$ in GCM, which should give a reasonable dynamical gluon mass.

Neglecting the four quark condensate contribution, the gluon pair condensate from the ID model takes the simple form

$$
g^{2}\left\langle A^{2}\right\rangle=2 N_{c} \eta^{2}
$$

In the case $\eta^{2} / 2=m_{\rho}^{2}=0.59 \mathrm{GeV}^{2}\left[23\right.$, the gluon pair condensate takes the value $7.1 \mathrm{GeV}^{2}$ and the corresponding gluon mass is $0.82 \mathrm{GeV}$ which is also in agreement with the estimates from other methods.

In Table 1, the magnitude of the obtained dimension 4 mixed condensate is much smaller in comparison with the recent results based on the quenched lattice results for the quark propagator in Landau gauge [4. Within the ID model with $\eta^{2} / 2=0.59 \mathrm{GeV}^{2}$, the mixed condensate takes the value $-0.0003 \mathrm{GeV}^{4}$. However, the magnitude of the quark condensate $-\langle\bar{q} q\rangle^{1 / 3}$ obtained in ID model with the same parameter is $118 \mathrm{MeV}$ which is also smaller in comparison with the standard value $250 \mathrm{MeV}$. If we simply lift the the value of $\eta$, for example, taking $\eta=1.8 \mathrm{GeV}$, we get that $g\langle\bar{q} A \cdot \gamma q\rangle=-0.0018 \mathrm{GeV}^{4}$ with $-\langle\bar{q} q\rangle^{1 / 3}=184 \mathrm{MeV}$. Therefore, the mixed condensates obtained in both the ID and Gaussian models are at the same order of magnitude. According to [1, 12, 4, the coefficient of $1 / Q^{4}$ in the expansion of the vector quark self-energy function at sufficiently large values of $Q$ is determined by the combined dimension 4 condensates

$$
\alpha_{s}\langle\bar{q} g A \cdot \gamma q\rangle-\frac{4 \pi}{27}\left\langle\frac{\alpha_{s}}{\pi} G^{2}\right\rangle,
$$

where the gluon condensate takes the value $\left\langle\frac{\alpha_{s}}{\pi} G^{2}\right\rangle \simeq 0.01 \mathrm{GeV}^{4}$. Multiplying $\alpha_{s}(\mu) \simeq 0.5$ to the mixed condensate where $\mu$ stands for a typical hadronic mass, we find that $\alpha_{s}\langle\bar{q} g A \cdot \gamma q\rangle$ is around $-0.0007 \mathrm{GeV}^{4}$ in our calculation. In comparison with the magnitude of $\left\langle\frac{\alpha_{s}}{\pi} G^{2}\right\rangle$, we find that the main contribution to Eq.(27) is determined by the gluon condensate. This result is in contrary to the one obtained in Ref [4. The obtained value for $\alpha_{s}\langle\bar{q} g A \cdot \gamma q\rangle$ in Ref [4] is $(-0.11 \pm 0.03) \mathrm{GeV}^{4}$, comparing to which the gluon condensate contribution to (27) can be neglected.

Table 2. The comparison of the contributions to the gluon pair condensate from the effective gluon propagator and the four quark condensate in Eq.(16).

\begin{tabular}{ccccc}
\hline \hline$\Delta(\mathrm{GeV})$ & $\omega(\mathrm{GeV})$ & $g^{2}\langle A A\rangle_{1}\left(\mathrm{GeV}^{2}\right)$ & $g^{2}\langle A A\rangle_{2}\left(\mathrm{GeV}^{2}\right)$ & $M_{g 1}(\mathrm{GeV})$ \\
\hline 30.0 & 0.40 & 9.22 & 0.28 & 0.93 \\
\hline 19.0 & 0.45 & 9.35 & -0.13 & 0.94 \\
\hline 13.2 & 0.50 & 9.90 & -0.05 & 0.96 \\
\hline \hline
\end{tabular}

According to the quark motion equation $\left(\gamma_{\mu} \partial_{\mu}+m-i \frac{\lambda^{a}}{2} A_{\mu}^{a} \gamma_{\mu}\right) q=0$, we expect that the 
condensate $\left\langle\bar{q}\left(\gamma_{\mu} \partial_{\mu}+m-i \frac{\lambda^{a}}{2} A_{\mu}^{a} \gamma_{\mu}\right) q\right\rangle$ should take zero. In the chiral limit, this relation is reduced to

$$
-i\left\langle\bar{q} \frac{\lambda^{a}}{2} A_{\mu}^{a} \gamma_{\mu} q\right\rangle=-\left\langle\bar{q} \gamma_{\mu} \partial_{\mu} q\right\rangle
$$

Actually, the evaluation of the Landau gauge condensate $\left\langle\bar{q} \gamma_{\mu} \partial \mu q\right\rangle$ is very simple in GCM. Repeating the procedure above, including the subtraction, we get

$$
\begin{aligned}
-\left\langle\bar{q}(x) \gamma_{\mu} \partial_{\mu} q(x)\right\rangle & =\left.\partial_{\mu}^{x^{\prime}}\left\{\operatorname{tr}\left[G\left(x^{\prime}-x\right) \gamma_{\mu}\right]-\operatorname{tr}\left[G^{\prime}\left(x^{\prime}-x\right) \gamma_{\mu}\right]\right\}\right|_{x^{\prime}=x} \\
& =12 \int \frac{d^{4} p}{(2 \pi)^{4}}\left\{\left[\frac{A\left(p^{2}\right) p^{2}}{A^{2}\left(p^{2}\right) p^{2}+B^{2}\left(p^{2}\right)}\right]-\left[\frac{1}{A^{\prime}\left(p^{2}\right)}\right]\right\} \\
& =\frac{3}{4 \pi^{2}} \int_{0}^{\infty} d s s\left[\frac{A(s) s}{A^{2}(s) s+B^{2}(s)}-\frac{1}{A^{\prime}(s)}\right] .
\end{aligned}
$$

It can been see that this is just the Eq.(19). However, without the subtraction, Eq.(19) reduces to

$$
-\frac{3}{4 \pi^{2}} \int_{0}^{\infty} d s s\left[\frac{B^{2}(s)}{A^{2}(s) s+B^{2}(s)}+\frac{A(s)(A(s)-1) s}{A^{2}(s) s+B^{2}(s)}\right],
$$

which is negative and divergent since $A(s)>1$ for finite $s$, while Eq.(29) reduces to

$$
\frac{3}{4 \pi^{2}} \int_{0}^{\infty} d s s\left[\frac{A(s) s}{A^{2}(s) s+B^{2}(s)}\right],
$$

which is positive and divergent. Therefore, there exists a large difference between these two expressions even in sign if we do not adopt the subtraction mechanism. This shows that it is reasonable to introduce the subtraction mechanism in our definition. For the massive case, according to the PCAC relation, the value of $m\langle\bar{q} q\rangle$ is around $-0.00008 \mathrm{GeV}^{4}$, which suggests that the Landau gauge dimension 4 mixed condensate is an order of magnitude larger than the $m\langle\bar{q} q\rangle$ condensate.

Here we still want to stress that the discrepancy of the estimates of the mixed quark gluon condensate arises from the different definitions of condensates. In Ref [4] and those related to it, the condensates are defined as the coefficients of expansion terms in A(s). Actually, by taking the same definition, similar results can also be obtained within Global Color Model. Consider the ID model described by Eqs.(22) and (23)

$$
A(s)=\frac{1}{2}\left[1+\sqrt{1+2 \eta^{2} / s}\right] \stackrel{\text { large }}{=}-s \text {. } 1+\frac{\eta^{2}}{2} \frac{1}{s}-\left[\frac{\eta^{2}}{2}\right]^{2} \frac{1}{s^{2}}+\cdots
$$

With the conventions of Ref. [4] one would read

$$
\begin{array}{r}
\alpha_{s}\left\langle A^{2}\right\rangle=\frac{N_{c}}{\pi} \frac{\eta^{2}}{2}, \\
\alpha_{s}\langle\bar{q} g A \cdot \gamma q\rangle-\frac{4}{9 N_{c}}\left\langle\frac{\alpha_{s}}{\pi} G^{2}\right\rangle=\frac{4}{3 \pi}\left[\frac{\eta^{2}}{2}\right]^{2} .
\end{array}
$$


Note that Eq.(33) is identical to Eq.(26). Substituting $\eta^{2} / 2=0.59 \mathrm{GeV}^{2}$ into Eqs.(33) and (34) one gets

$$
\begin{aligned}
\alpha_{s}\left\langle A^{2}\right\rangle & =0.57 \quad \mathrm{GeV}^{2}, \\
\alpha_{s}\langle\bar{q} g A \cdot \gamma q\rangle-\frac{4}{9 N_{c}}\left\langle\frac{\alpha_{s}}{\pi} G^{2}\right\rangle & =-0.15 \quad \mathrm{GeV}^{2},
\end{aligned}
$$

which are in agreement with the results of Ref. 4 .

The small value of the mixed quark gluon condensate obtained based on the definition (12) can be tracked back to subtracting the coefficients $1 / s$ and $1 / s^{2}$ in the expansion of $A^{\prime}(s)$ evaluated in the Wigner phase from those obtained with $A(s)$ calculated in the Nambu phase. In the case of sufficiently large value of $\mathrm{s}$

$$
A(s)=1+c_{1}^{N} \frac{1}{s}+c_{2}^{N} \frac{1}{s^{2}} \quad, \quad A^{\prime}(s)=1+c_{1}^{W} \frac{1}{s}+c_{2}^{W} \frac{1}{s^{2}},
$$

one gets

$$
\alpha_{s}\langle\bar{q} g A \cdot \gamma q\rangle-\frac{4}{9 N_{c}}\left\langle\frac{\alpha_{s}}{\pi} G^{2}\right\rangle=c_{2}^{N}-c_{2}^{W} .
$$

The fact that the vector self-energy function of the quark propagator is not too sensitive to the phase in large momentum, i.e., $c_{2}^{N} \approx c_{2}^{W}$, can qualitatively explain why the 1.h.s of Eq.(38) is small according to our definition.

\section{Discussion and conclusion}

We have evaluated the gluon pair condensate and the dimension 4 mixed quark gluon condensate in Landau Gauge within the framework of GCM. To avoid divergence and subtract the perturbative contribution, two measures are taken in our calculations. The input effective gluon propagator adopted in our calculation has finite range in momentum space, which can suppress the divergence induced by the UV behavior of the scalar quark self-energy function. This is reasonable since the condensates are mainly determined by the low energy properties of QCD. To subtract the perturbative contribution, the trivial solution or the Wigner solution to the quark DSE was used self-consistently in our formalism.

With above method, the contributions to the gluon pair condensate was decomposed into two parts: the contribution from the effective gluon propagator and the contribution from the four quark condensate. From our numerical study, we find that the correction to the gluon pair condensate from the four quark condensate is very small and can be neglected. The obtained gluon pair condensate and the corresponding dynamical gluon mass are compatible with other estimations in the literatures. The expression for the dimension 4 mixed quark gluon condensate is very simple in our formalism. This expression has been verified by using the quark motion equation. The value of the mixed condensate $\langle g \bar{q} A \cdot \gamma q\rangle$ is an order of magnitude larger than the condensate $m\langle\bar{q} q\rangle$. 
We also explicated that the discrepancy of the estimates of the Landau mixed quark gluon condensate between our formalism and others arises from the different definitions of the condensates. If we take the same definition as in Ref. 4, the obtained values of both the gluon pair condensate and the mixed condensate in GCM are consistent with the corresponding ones extracted from Lattice simulation results. That means when the same definitions are used, the GCM/DSE formalism is completely consistent with the analysis based on the lattice results. Note that due to the ambiguity in the definitions of the condensates, there exists no natural or unique definitions but only clear definitions; when the same definitions are used, all wellconstrained models should yield the same result. This fact is made very clear for the quark condensate $\langle\bar{q} q\rangle[26]$.

\section{References}

[1] M. J. Lavelle and M. Schaden, Phys. Lett. B208 (1988) 297.

[2] P. Boucaud, A. Le Yaouanc, J. P. Leroy, J. Micheli, O. Pene and J. Rodriguez-Quintero, hep-ph/0101302, Phys. Rev. D63 (2001) 114003.

[3] P. Boucaud, A. Le Yaouanc, J. P. Leroy, J. Micheli, O. Pene and J. Rodriguez-Quintero, Phys. Lett. B493 (2000) 315. P. Boucaud, G. Burgio, F. Di Renzo, J. P. Leroy, J. Micheli, C. Parrinello, O. Pene, C. Pittori, J. Rodriguez-Quintero, C. Roiesnel and K. Sharkey, JHEP 04 (2000) 006.

[4] E. R. Arriola, P. O. Bowman and W. Broniowski, hep-ph/0408309, Phys. Rev. D70 (2004) 097505.

[5] L. S. Celenza and C. M. Shakin, Phys. Rev. D34 (1986) 1591.

[6] K. I. Kondo, Phys. Lett. B514 (2001) 335.

[7] K. I. Kondo, Phys. Lett. B572 (2003) 210.

[8] A. A. Slavov, hep-ph/0407194.

[9] F. V. Gubarev, L. Stodolsky and V. I. Zakharov, hep-ph/0010057, Phys. Rev. Lett. 86(2001) 2220 .

[10] F. V. Gubarev and V. I. Zakharov, hep-ph/0010096, Phys. Lett. B501 (2001) 28.

[11] V. P. Spiridinov, Mod. Phys. Lett. A5 (1990) 653; A6 (1991) 277.

[12] M. J. Lavelle and M. Oleszczuk, Phys. Lett. B275 (1992) 133. 
[13] R. T. Cahill and S. M. Gunner, hep-ph/9812491, Fizika B7 (1998) 171.

[14] P. C. Tandy, Prog. Part. Nucl. Phys. 39 (1997) 117, and reference therein.

[15] C. D. Roberts and A. G. Villianms, Prog. Part. Nucl. Phys. 33 (1994) 477, and referrence therein.

[16] D. Diakonov, M. V. Polyakov abd C. Weiss, Nucl. Phys. B461 (1996) 539; M. V. Polyakov abd C. Weiss, Phys. Lett. B387 (1996) 841.

[17] T. Meissner Phys. Lett. B405 (1997) 8.

[18] K. D. Lane, Phys. Rev. D 10 (1974) 2605; H. D. Politzer, Nucl. Phys. B 117 (1976) 397.

[19] P. Maris, C. D. Roberts and P. C. Tandy, Phys. lett. B420 (1998) 267; P. Maris and C. D. Roberts, Phys. Rev. C 56 (1997) 3369.

[20] Z. Zhang and W. Q. Zhao, Phys. Lett. B610 (2005) 235.

[21] Z. Zhang and W. Q. Zhao, hep-ph/0409100

[22] D. Blaschke, C. D. Roberts and S. Schimidt, Phys. Lett. B 425 (1998) 232-238. D. Blaschke and C. D. Roberts, Nucl. Phys. A624 (1998) 179c-209c. D. Blaschke, H. Grigorian, G. Poghosyan, C. D. Roberts, S.Schmidt, Phys. lett. B 450 (1999) 207-214.

[23] H. J. Munczek and A. M. Nemirovsky, Phys. Rev. D28 (1983) 181.

[24] R. Alkofer, P. Watson and H. Weigel, Phys. Rev. D 65 (2002) 094026; H. Weigel, R. Alkofer and P. Watson, hep-ph/0307066.

[25] J. H. Field, hep-ph/0101158, Phys. Rev. D66 (2002) 013013.

[26] K. Langfeld, H. Markum, R. Pullirsch, C. D. Roberts and S. M. Schmidt, Phys. Rev. C 67(2003) 065206. 